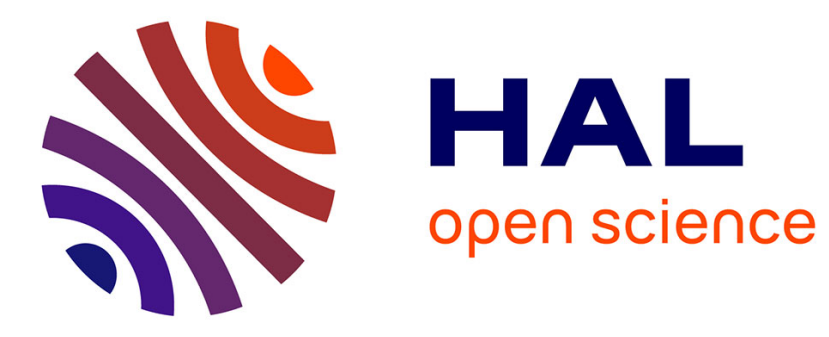

\title{
Spectroscopic Measurement of Interlayer Screening in Multilayer Epitaxial Graphene
}

Dong Sun, Charles Divin, Claire Berger, Walt A. de Heer, Philip N. First, Theodore B. Norris

\section{- To cite this version:}

Dong Sun, Charles Divin, Claire Berger, Walt A. de Heer, Philip N. First, et al.. Spectroscopic Measurement of Interlayer Screening in Multilayer Epitaxial Graphene. Physical Review Letters, 2010, 104 (13), pp.136802. 10.1103/PhysRevLett.104.136802 . hal-00911815

\section{HAL Id: hal-00911815 \\ https://hal.science/hal-00911815}

Submitted on 30 Nov 2013

HAL is a multi-disciplinary open access archive for the deposit and dissemination of scientific research documents, whether they are published or not. The documents may come from teaching and research institutions in France or abroad, or from public or private research centers.
L'archive ouverte pluridisciplinaire HAL, est destinée au dépôt et à la diffusion de documents scientifiques de niveau recherche, publiés ou non, émanant des établissements d'enseignement et de recherche français ou étrangers, des laboratoires publics ou privés. 


\title{
Spectroscopic Measurement of Interlayer Screening in Multilayer Epitaxial Graphene
}

\author{
Dong Sun, ${ }^{1}$ Charles Divin, ${ }^{1}$ Claire Berger, ${ }^{2}$ Walt A. de Heer, ${ }^{2}$ Phillip N. First, ${ }^{2}$ and Theodore B. Norris ${ }^{1, *}$ \\ ${ }^{1}$ Center for Ultrafast Optical Science, University of Michigan, Ann Arbor, Michigan 48109-2099, USA \\ ${ }^{2}$ School of Physics, Georgia Institute of Technology, Atlanta, Georgia 30332, USA
}

(Received 8 September 2009; published 1 April 2010)

\begin{abstract}
The substrate-induced charge-density profile in carbon face epitaxial graphene is determined using nondegenerate ultrafast midinfrared pump-probe spectroscopy. Distinct zero crossings in the differential transmission spectra are used to identify the Fermi levels of layers within the multilayer stack. Probing within the transmission window of the $\mathrm{SiC}$ substrate, we find the Fermi levels of the first four heavily doped layers to be, respectively, 360, 215, 140, and $93 \mathrm{meV}$ above the Dirac point. The charge screening length is determined to be one graphene layer, in good agreement with theoretical predictions.
\end{abstract}

PACS numbers: 73.90.+f, 78.67.Pt

Graphene grown epitaxially on $\mathrm{SiC}$ substrates is a promising platform for carbon-based nanoelectronics and molecular electronics [1,2]. High-quality multilayer graphene is grown via thermal decomposition of the carbonterminated surface of SiC. Accumulating evidence indicates that the resulting structure behaves as multilayer graphene and not graphite [3-9]. From transport measurements, the layer closest to the substrate is known to be electron doped, with the density dropping rapidly for subsequent layers [10]. A key question to be addressed for the development of epitaxial graphene devices is, What is the actual charge-density profile of the layers? Dielectric screening in a two- dimensional electron gas has been of fundamental physics interest since the 1970s [11-14]. From a device engineering point of view, a determination of exact charge-density profiles and interlayer screening effects in stacked graphene layers is required to understand the electric field effect for future nanoelectronic devices based on multilayer epitaxial graphene [1].

Several approaches have been taken to address the question of interlayer screening in multilayer epitaxial graphene. The highly conducting layer has been characterized by electronic transport measurements [10], and Landau level spectroscopy was used to study the nearly neutral layers on the top $[6,7]$. These experiments determine, respectively, the charge density close to the $\mathrm{SiC}$ substrate and the charge density far from the substrate. Measurement of the layer-to-layer variation of charge density (or potential) has been accomplished in related systems. The layer-dependent band structure and charge density of 1-4 graphene layers grown on $\mathrm{SiC}(0001)$ ( $\mathrm{Si}$ face) has been found by angle-resolved photoemission spectroscopy $[15,16]$, and scanning probe methods have been applied to measure surface potentials in few-layer graphene flakes [17] and in epitaxial graphene on $\mathrm{SiC}(0001)$ [18]. However, these measurements are only sensitive to the top layers. A direct measurement of charge density or potential variation within the buried near-substrate layers of thick multilayer graphene has not been available.
The charge density of the first few layers in epitaxial graphene is caused by the built-in electric field at the SiCgraphene interface $[2,19]$. Strong covalent bonds exist between the substrate and the first graphitic interface layer, which is devoid of graphene electronic properties and is denoted as layer number zero in this Letter to distinguish it from the first heavily doped graphene layer [19]. The graphene-SiC charge transfer apparently does not rely on doping of the $\mathrm{SiC}$ substrate; it originates from the $\mathrm{SiC}$ and graphene interface only [16].

The theoretical problem of the charge distribution in a stack of 2D electron gases was first considered in 1971 [11] for parabolic bands with no interlayer coupling. Interlayer hopping modifies the picture. For Bernal-stacked (graphitic) multilayers, Guinea [20] predicts a charge screening length of 2-3 graphene layers, with significant charge oscillations between alternate layers. Related experiments using a graphite-channel field-effect transistor found a charge screening length of 3-4 graphene layers [21]. A physical model closer to epitaxial graphene, with rotational stacked layers under external field, has been discussed in the context of graphene bilayers only [22,23]. However, given existing experimental indications of noninteracting layers, a nonlinear Thomas-Fermi model of uncoupled graphene sheets [17] would appear to be appropriate to describe interlayer screening in multilayer epitaxial graphene. In that model, a power-law potential decay is predicted, with no oscillatory component [17].

In this Letter, we present an optical nondegenerate pump-probe measurement that determines the charge densities of the first four layers in multilayer epitaxial graphene. The 63-layer sample (thickness determined by ellipsometry) is produced by thermal desorption of $\mathrm{Si}$ from the C-terminated face of single-crystal $4 \mathrm{H}-\mathrm{SiC}(000 \overline{1})$, as described elsewhere [2]. The principle of the experiment is well established [24], but perhaps not broadly known. Therefore, we present a simple schematic of the measurement in Fig. 1: An 800-nm ultrafast pump pulse excites quasiparticles high into the conduction band, where fast electron-electron scattering establishes a hot 
thermal distribution within a few tens of femtoseconds. Subsequent equilibration with the lattice is initially dominated by high energy optical phonons, while cooling via acoustic phonons occurs for times of order 1-10 ps. During the lattice-cooling period, the transient occupation probability above (below) the Fermi energy is larger (smaller) than before the pump; thus, the change in probe transmission induced by the pump [differential transmission (DT)] is positive (negative) for probe transitions above (below) the Fermi energy in each doped graphene layer. For undoped layers, the DT signal is always slightly positive because all probe photon energies correspond to interband transitions above the Fermi energy $\left(E_{F}\right.$; we take the Dirac point energy to be zero). For this work, the probe transitions lie far into the tails of the transient hot-electron distribution in the undoped layers; consequently, the positive DT background is small, even for $\sim 60$ undoped layers. However, when the probe photon energy approaches $2 E_{F}$ for any single doped layer, the DT at a fixed probe delay will become strongly negative, dominating the positive background. At photon energies slightly above $2 E_{F}$, the DT will switch to strongly positive. Because this transition is abrupt in energy, the influence of the positive background is minimal, and $2 E_{F}$ for this layer lies essentially at the zero-crossing energy. A scan of DT versus probe energy (fixed delay) can be used to identify $E_{F}$ for each doped layer lying within the scanned energy range. These ideas will be verified by simulations shown later. We also note that there is an open question as to whether electronelectron scattering eliminates holes within the first $100 \mathrm{fs}$ [25], but this does not affect the main results of this Letter because the high energy probe photon is not sensitive to holes that relax to the vicinity of Dirac point.

Figure 2(a) shows a schematic of the multilayer graphene sample and $\mathrm{SiC}$ substrate. Energy diagrams at the right depict the qualitative profile of layer charge densities. In Ref. [24], we showed that the position of the Fermi level of the most highly doped layer of the sample (layer 1) could be determined from a zero crossing in the DT spectrum. Here we extend the spectral range of the probe to longer wavelengths, thus enabling us to determine the charge density in each of the first four monolayers of a multilayer sample. Because the Fermi levels of the layers are sufficiently well separated, we are able for the first time

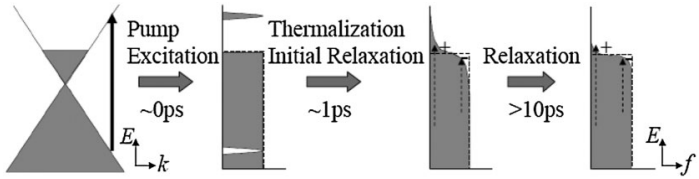

FIG. 1. Nonequilibrium distribution of hot carriers following pump excitation. On the left is the graphene band structure for a doped layer; optical excitation is indicated by the vertical solid arrow. The carrier distribution functions at time delays of 0,1 , and $>10 \mathrm{ps}$ are schematically shown; the optical probe is indicated by vertical dashed arrows, and the "+" and "-" indicators mark the sign of the resulting DT signal. to obtain a quantitative measurement of the interlayer screening length in this multilayer system.

In our experimental implementation, the pump pulse ( $70 \mathrm{fs}$ at $800 \mathrm{~nm}$ using a $250-\mathrm{kHz}$ regenerative amplifier) and the probe are both incident from the graphene side of the sample. The midinfrared probe pulse is generated from a tunable femtosecond optical parametric amplifier (OPA, 1.1-1.6 $\mu \mathrm{m}$ signal and 1.6-2.5 $\mu \mathrm{m}$ idler with pulse width $\sim 150 \mathrm{fs}$ ) or a differential frequency generator (DFG, 2.6-10 $\mu \mathrm{m}$ with pulse width $\sim 220 \mathrm{fs}$ ). Pump and probe beams are collinearly polarized and focused on the sample to $\sim 80$ and $40 \mu \mathrm{m}$ diameter for the OPA probe or $\sim 150$ and $\sim 80 \mu \mathrm{m}$ for the DFG probe. Probe wavelengths from 1.2 to $7 \mu \mathrm{m}$ are scanned except for inaccessible regions between 2.5 and $2.7 \mu \mathrm{m}$ (OPA to DFG gap), and between 5.8 and $6.7 \mu \mathrm{m}$ (due to multiphoton absorption in the $\mathrm{SiC}$

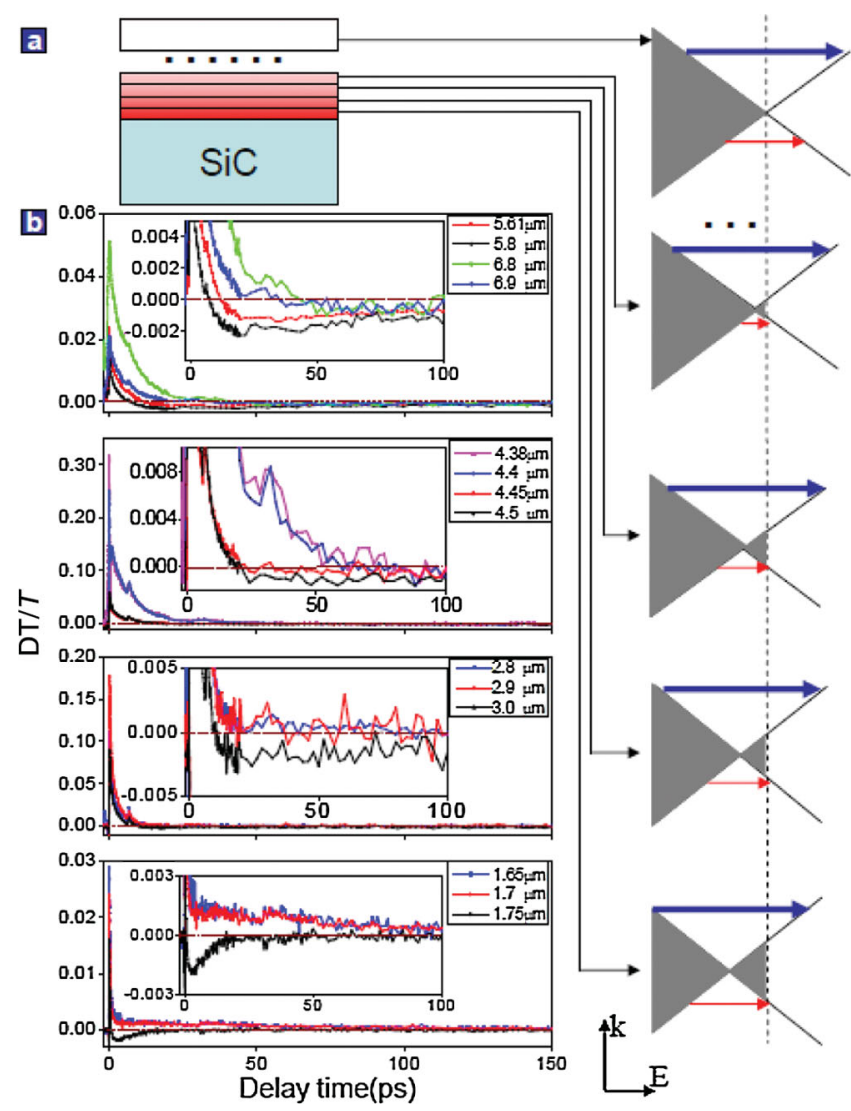

FIG. 2 (color). Sample structure and DT zero crossings. (a). Schematic diagram of sample structure, energy dispersion, and Fermi levels of the graphene layers; the top right figure indicates the band structure for the multiple undoped layers; each of the four doped layers are shown in order below (most heavily doped layer nearest the substrate). The Fermi level is labeled with a dashed line (black). The blue solid line shows the transitions induced by the 800-nm optical pump pulse; the red lines correspond to probe transitions around the DT/ $T$ zero crossings. (b). Time scans of different probe wavelengths around four DT zero crossings. In the insets the DT $/ T$ relaxation tails are shown expanded around zero DT $/ T$. The horizontal dashed lines (brown) mark where the DT/ $T$ is zero. The 800 -nm pump power is $1,9,9$, and $1 \mathrm{~mW}$, respectively, from top to bottom. 
substrate; this also imposes the 7- $\mu \mathrm{m}$ cutoff [26]). The DT signal from the $\mathrm{SiC}$ substrate contributes only to the timezero signals (within the pulse width) and is typically 100 times smaller than the graphene DT signal. The transmission of both pump and probe beams through the 63layers sample is about $30 \%$, which corresponds to the expected 2.3\% absorption in each graphene layer [27]. Most experiments are performed using $1 \mathrm{~mW}$ pump power, corresponding to $2 \times 10^{12}$ photon $/ \mathrm{cm}^{2}$ on layer 1 considering the multiple-layer absorption. The probe beam beyond the sample is spatially filtered in a monochromator and detected by either an InGaAs photodetector or liquid nitrogen cooled $\mathrm{HgCdTe}$ or InSb photodetector. Synchronous acquisition is done via a lock-in amplifier referenced to the $4.2-\mathrm{kHz}$ mechanically chopped pump. All experiments discussed here are performed at $10 \mathrm{~K}$ in a helium flow cryostat.

Figure 2(b) shows the fractional change in transmission induced by the pump (DT/T) as a function of probe delay time for probe wavelengths near four DT zero crossings. The bottom plot shows that beyond $\sim 10 \mathrm{ps}$ the DT changes sign at a wavelength between 1.7 and $1.75 \mu \mathrm{m}$, which gives $E_{F}$ between 355 and $365 \mathrm{meV}$ for the most heavily doped layer. This nominal value varies by less than $10 \%$ for different positions on the sample. Proceeding upwards, the second plot in Fig. 2(b) shows DT reverting to positive around $2.7 \mu \mathrm{m}$ and back to negative at $3.0 \mu \mathrm{m}$, indicating a Fermi energy between 207 and $222 \mathrm{meV}$. We attribute this second zero crossing to a doped graphene layer farther from the $\mathrm{SiC}$ interface — and not to a separate domain-because of the small energy variation found for the most heavily doped layer. A third DT zero crossing is found between 4.38 and $4.45 \mu \mathrm{m}\left(E_{F}\right.$ between 139 and $142 \mathrm{meV}$ ) as shown in the third plot of Fig. 2(b). There are strong indications of a fourth zero crossing near $7 \mu \mathrm{m}$ [Fig. 2(b), top], but the background is imperfectly determined due to $\mathrm{SiC}$ absorption (differential reflection may be used in the future). Allowing for a small negative background shift (note the DT/T long-time asymptote), the fourth zero crossing is estimated to be around $6.9 \mu \mathrm{m}$.

Although the intuitive picture given in Fig. 1 illustrates how thermal smearing of the population gives rise to a sign change in differential transmission, it neglects the possible contribution of pump-induced changes in the reflectivity of the sample. Therefore, to connect the DT spectra to population changes more rigorously, we model the optical response of the multilayer graphene system using the transfer matrix method. The transfer matrix of a layer relates the incident and reflected fields via the dynamical conductivity of the layer; we utilize a model for the conductivity including its dependence on electron temperature and layer charge density [28]. The product of all transfer matrices, including that of the substrate, gives the optical response of the multilayer epitaxial graphene sample. In the simulation we include four highly doped layers with charge densities corresponding to those measured in the experiment. For simplicity, we use a common electron temperature in all graphene layers at all probe delays. Although the transient carrier distributions of different graphene layers may not be in strict equilibrium with one another, this will not affect the DT zero-crossing energies, which depend on only the corresponding layer doping.
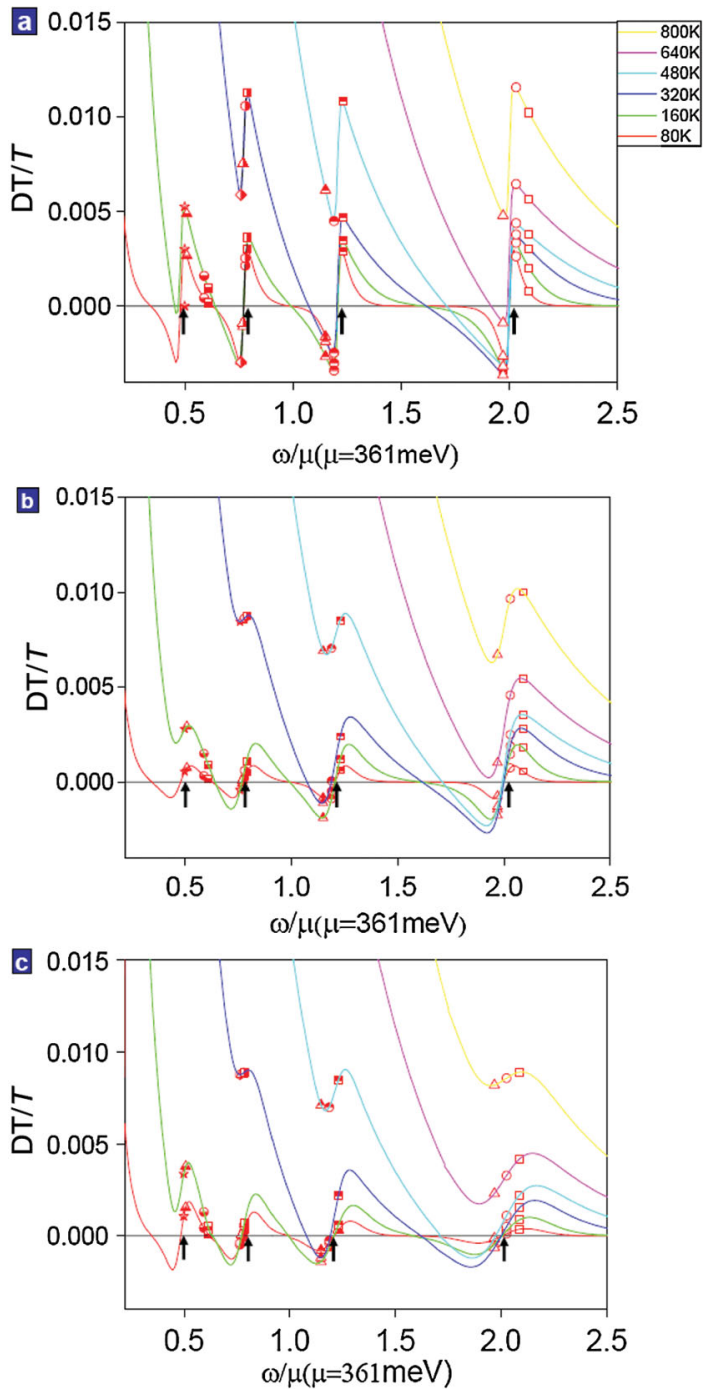

FIG. 3 (color). DT signal simulation. (a) Simulated DT $/ T$ spectra from the transfer matrix model with lattice temperature at $10 \mathrm{~K}$ and no inhomogeneous broadening. (b) Simulated DT/T spectra with lattice temperature at $50 \mathrm{~K}$ and no inhomogeneous broadening. (c) Simulated DT $/ T$ curves with lattice temperature at $10 \mathrm{~K}$ and a $10 \%$ inhomogeneous broadening of the Fermi level. All simulations include the multiple undoped layers as well as the four heavily doped layers with Fermi levels extracted from the experiment; the black arrows indicate the probe photon energy at the DT zero crossings corresponding to the observed Fermi levels. The probe photon energies of the DT scans in Fig. 2 are indicated on the simulated DT spectra with the different symbol styles around each zero crossing. For example, around $\omega / \mu=2$, the empty square corresponds to $1.65 \mu \mathrm{m}$, empty circle corresponds to $1.7 \mu \mathrm{m}$, and empty triangle corresponds to $1.75 \mu \mathrm{m}$, respectively, for the curves at the bottom plot of Fig. 2(b). All the figures share the same legend. 


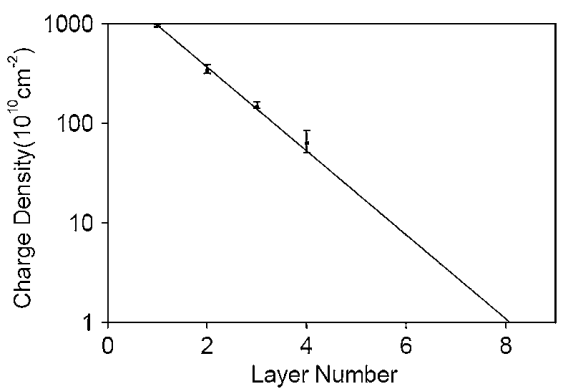

FIG. 4. Screening length fitting. Exponential fit to the experimental charge density of the first four heavily doped layers.

Figure 3(a) shows the simulation results for a sample ambient temperature of $10 \mathrm{~K}$. It is easy to see that the qualitative behavior of DT versus probe energy follows from the discussion of Fig. 1. Nevertheless, there is a significant discrepancy between this simulation and the experimental results: the slope of the calculated DT curve $[\Delta(\mathrm{DT} / T) / \Delta \omega]$ around the zero crossings is steeper than that measured in the experiment [24]. Possible reasons for the discrepancies are a somewhat elevated background lattice temperature, and inhomogeneous broadening due to sample nonuniformity. The actual lattice temperature is not measured directly since there is an unknown temperature gradient between the cryostat temperature sensor and the graphene sample. Figure 3(b) shows the simulation for a background (i.e., unpumped) electron temperature of $50 \mathrm{~K}$. The positive DT signal at low electron temperature decreases significantly and the slope at zero crossings lessens with increasing lattice temperature. Inhomogeneous broadening can arise from potential variations at the $\mathrm{SiC}$ interface. A typical intrinsic disorder length scale has been measured to be several $\mu \mathrm{m}$ in exfoliated graphene and epitaxial graphene, respectively [5,29], which is below our probe spot size. Since position-dependent variations of the zero-crossing energy were observed to be under $10 \%$, we show in Fig. 3(c) the simulated DT spectrum with a background electron temperature of $10 \mathrm{~K}$, including a Gaussian distribution of $E_{F}$ values with full width at half maximum equal to $0.10 E_{F}$. This $10 \%$ inhomogeneous broadening converts to $36 \mathrm{meV}$ or $420 \mathrm{~K}$ for the most heavily doped layers, which is smaller than the initial electron temperature (estimated to be above $1000 \mathrm{~K}$ immediately following the pump). Adding both effects brings the simulation closer to the experimental observations. However, the DT versus energy still crosses zero at $2 E_{F}$ for each layer.

Figure 4 shows the layer charge density $n=E_{F}^{2} / \pi \hbar^{2} v_{F}^{2}$ versus layer index on a semilog plot. Error bars correspond to the $E_{F}$ ranges quoted above (for the fourth doped layer, we conservatively set the low energy limit at $7.5 \mu \mathrm{m}$, beyond the scanning range). The solid curve is a fit to a simple exponential decay: $n_{l}=n_{1} e^{-(l-1) / l_{s}}$, where $l$ is the graphene layer index and $n_{1}=9.56 \times 10^{12} \mathrm{~cm}^{-2}$ is the charge density of the first doped layer. The fit determines a charge screening length $l_{s}=1.02$ graphene layers (potential screening length 2.04 layers). Surprisingly, the mea- sured functional form of the screening is different from the power law predicted by a Thomas-Fermi model for uncoupled graphene planes [17], yet the potential screening length is comparable to a recent prediction for Bernalstacked layers [20].

In conclusion, we have used pump-probe spectroscopy to resolve the sheet charge-density profile of a multilayer epitaxial graphene sample and determined the charge screening length to be 1 layer. The measured DT spectrum can be well explained by a dynamic conductivity simulation when incorporating in-plane disorder and an elevated lattice temperature. A realistic theory for rotationally stacked multilayer graphene is expected to reconcile the measured "uncoupled" band structure with the newly observed exponential screening. For electronic devices, the short screening length implies that gate doping will be effective only for the layer adjacent to the gate electrode.

This project has been supported by NSF MRSEC 0820382.

*To whom all correspondence should be addressed. tnorris@eecs.umich.edu

[1] C. Berger et al., J. Phys. Chem. B 108, 19912 (2004).

[2] W. A. De Heer et al., Solid State Commun. 143, 92 (2007).

[3] X. Wu et al., Phys. Rev. Lett. 98, 136801 (2007).

[4] J. Hass et al., Phys. Rev. Lett. 100, 125504 (2008).

[5] M. Sprinkle et al., Phys. Rev. Lett. 103, 226803 (2009).

[6] M. L. Sadowski, Phys. Rev. Lett. 97, 266405 (2006); M. L. Sadowski et al., Solid State Commun. 143, 123 (2007).

[7] M. Orlita et al., Solid State Commun. 149, 1128 (2009); M. Orilta et al., Phys. Rev. Lett. 101, 267601 (2008).

[8] C. Faugeras et al., Appl. Phys. Lett. 92, 011914 (2008).

[9] D. L. Miller et al., Science 324, 924 (2009).

[10] C. Berger et al., Science 312, 1191 (2006).

[11] P. B. Visscher and L. M. Falicov, Phys. Rev. B 3, 2541 (1971).

[12] L. Pietronero et al., Phys. Rev. Lett. 41, 763 (1978).

[13] D. P. DiVincenzo and E. J. Mele, Phys. Rev. B 29, 1685 (1984).

[14] D. A. Dahl, Phys. Rev. B 37, 6882 (1988).

[15] T. Ohta et al., Phys. Rev. Lett. 98, 206802 (2007).

[16] S. Y. Zhou et al., Nature Mater. 6, 770 (2007).

[17] S. S. Datta et al., Nano Lett. 9, 7 (2009).

[18] P. Lauffer et al., Phys. Rev. B 77, 155426 (2008).

[19] F. Varchon et al., Phys. Rev. Lett. 99, 126805 (2007).

[20] F. Guinea, Phys. Rev. B 75, 235433 (2007).

[21] H. Miyazaki et al., Appl. Phys. Express 1, 034007 (2008).

[22] J. M. B. Lopes de Santos et al., Phys. Rev. Lett. 99, 256802 (2007).

[23] S. Shallcross et al., J. Phys. Condens. Matter 20, 454224 (2008).

[24] D. Sun et al., Phys. Rev. Lett. 101, 157402 (2008).

[25] J. M. Dawlaty et al., Appl. Phys. Lett. 93, 131905 (2008).

[26] J. M. Dawlaty et al., Appl. Phys. Lett. 92, 042116 (2008).

[27] R. R. Nair et al., Science 320, 1308 (2008).

[28] S. A. Mikhailov and K. Ziegler, Phys. Rev. Lett. 99, 016803 (2007).

[29] J. Martin et al., Nature Phys. 4, 144 (2008). 